\title{
Real and financial market interactions in a multiplier-accelerator model: Nonlinear dynamics, multistability and stylized facts
}

Cite as: Chaos 27, 103120 (2017); https://doi.org/10.1063/1.4994617

Submitted: 06 July 2017 . Accepted: 04 October 2017 . Published Online: 20 October 2017

F. Cavalli, A. Naimzada, and N. Pecora
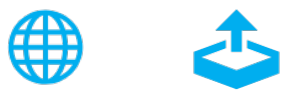

\section{ARTICLES YOU MAY BE INTERESTED IN}

\section{Attractors in complex networks}

Chaos: An Interdisciplinary Journal of Nonlinear Science 27, 103105 (2017); https:// doi.org/10.1063/1.4996883

\section{Opinion diversity and community formation in adaptive networks}

Chaos: An Interdisciplinary Journal of Nonlinear Science 27, 103115 (2017); https://

doi.org/10.1063/1.4989668

\section{Agents' beliefs and economic regimes polarization in interacting markets}

Chaos: An Interdisciplinary Journal of Nonlinear Science 28, 055911 (2018); https:// doi.org/10.1063/1.5024370

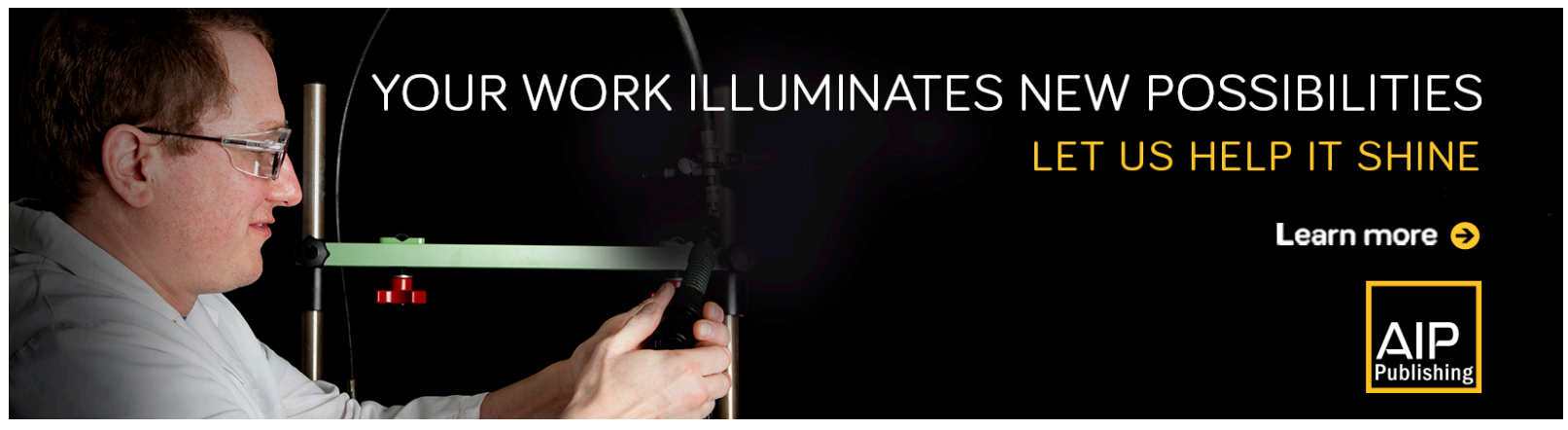




\title{
Real and financial market interactions in a multiplier-accelerator model: Nonlinear dynamics, multistability and stylized facts
}

\author{
F. Cavalli, ${ }^{1, a)}$ A. Naimzada, ${ }^{2, b)}$ and N. Pecora ${ }^{3, c)}$ \\ ${ }^{1}$ Department of Mathematical Sciences, Mathematical Finance and Econometrics, Catholic University, \\ Via Necchi 9, 20123 Milano, Italy \\ ${ }^{2}$ Department of Economics, Management and Statistics, University of Milano-Bicocca, U6 Building, \\ Piazza dell'Ateneo Nuovo 1, 20126 Milano, Italy \\ ${ }^{3}$ Department of Economics and Social Sciences, Catholic University, Via Emilia Parmense 84, \\ 29100 Piacenza, Italy
}

(Received 6 July 2017; accepted 4 October 2017; published online 20 October 2017)

\begin{abstract}
In the present paper, we investigate the dynamics of a model in which the real part of the economy, described within a multiplier-accelerator framework, interacts with a financial market with heterogeneous speculators, in order to study the channels through which the two sectors influence each other. Employing analytical and numerical tools, we investigate stability conditions as well as bifurcations and possible periodic, quasi-periodic, and chaotic dynamics, enlightening how the degree of market interaction, together with the accelerator parameter and the intervention of the fiscal authority, may affect the business cycle and the course of the financial market. In particular, we show that even if the steady state is locally stable, multistability phenomena can occur, with several and complex dynamic structures coexisting with the steady state. Finally, simulations reveal that the proposed model is able to explain several statistical properties and stylized facts observed in real financial markets, including persistent high volatility, fat-tailed return distributions, volatility clustering, and positive autocorrelation of absolute returns. Published by AIP Publishing.

https://doi.org/10.1063/1.4994617
\end{abstract}

The recent financial crisis unveiled and stressed how instability is an intrinsic feature of the overall economic environment, ranging from the real sector to the financial one. A growing literature has been investigating how speculative phenomena in financial markets are transmitted to the real economy and whether real market developments feed back on the financial sector. Similarly, an increasing interest concerned the phenomenon of financialization of the real economy, that is, the process by which the volume and the significance of financial instruments and contracts have grown relatively to the overall economic system. Since financialization transforms the functioning of the economic system at the macro level, it turns out to be relevant for the investigation of the two interacting subsystems in light of the recent economic developments. It is not news that macroeconomic variables, such as national income, employment rate, interest rate, and exchange rate, exhibit persistent and irregular fluctuations. Dynamical systems and chaos theory have been shedding some light on the roles of nonlinearity in deterministic processes to explain various complex dynamic behaviors of such variables. Our research goal is to deepen the understanding of the role of real and financial feedback mechanisms in order to identify, by means of rigorous analytical investigations complemented by numerical simulations, how instability may spread between the integrated markets.

\section{INTRODUCTION}

In his milestone contribution, Samuelson (1939) proposed the multiplier-accelerator model, which actually gave birth to the modern business cycle theory and to a wide research strand, focusing on the study of the real economy dynamics. One of the main novelties of Samuelson (1939) was to show that, combining the principle of accelerator and the multiplier analysis, fluctuations in the economic activity can endogenously arise as a consequence of the interplay between consumptions, investments, and national income changes. But one of the main drawbacks of the original framework of Samuelson (1939) was the lack of long-lasting economic cycles. To overcome this issue, several improvements have been proposed, for example, by considering the monetary sector or the inventory adjustment (see Day and Chen, 1993 and Hommes, 1995) as well as bounded investment functions (see Hicks, 1950; Puu et al., 2005; and Naimzada and Pecora, 2017).

Besides this historical evolution of the multiplieraccelerator framework, which enhanced the explanation of business cycle dynamics, the recent 2008-2009 crisis unveiled, once more, how instability is an intrinsic feature of the overall economic environment, ranging from the real sector to the financial one. After the facts occurred with the crisis, a growing literature has been investigating how speculative phenomena in financial markets are transmitted to the real economy and whether real market developments feed back into the financial sector. With respect to this, we mention the contributions by Charpe et al. (2011), who combines a financial setting characterized by heterogeneous interacting

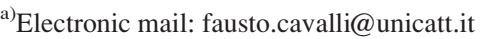

b)Electronic mail: ahmad.naimzada@unimib.it

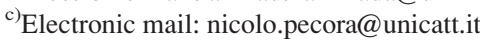


agents with a Tobin-like portfolio approach, and by Westerhoff (2012), who adopts a Keynesian good market approach taking into account a stock market with heterogeneous speculators. Additionally, Naimzada and Pireddu (2015) consider the evolutionary selection of forecasting rules performed by heterogeneous speculators when interacting real and financial markets are considered. It is worth noticing that when integrated real and financial sectors are studied, one must identify the channels through which the influence between the two sectors is realized. It is debatable which channels are the most relevant ones. The works in Naimzada and Pireddu (2014); Chen et al., (2012); Lengnick and Wohltmann (2013); and Westerhoff (2012) contain examples to describe the repercussions from the financial market to the real sector through channels such as wealth effects or collateral-based cost effects. On the other side, the opposite influence direction can be supported too, as the works of Naimzada and Pireddu (2014); Lengnick and Wohltmann (2013); Bask (2012); and Kontonikas and Montagnoli (2006) highlight.

The present paper belongs to the stream of literature which deals with the interactions between real and financial markets. Our research is also motivated by the so-called phenomenon of financialization of the real economy, that is, an expansion in the size and importance of the financial sector with respect to the overall economic system. Accordingly, this results in raising the significance of the financial sector with respect to the real one and in transferring income from the real to the financial sector. Financialization operates through different channels as well: changes in the skeleton and operativeness of financial markets, in the behavior of non-financial institutions and in economic policy. Since financialization transforms the functioning of the economic system at the macro level, it turns out to be relevant for the investigation of the two interacting subsystems in light of the recent economic developments.

Concerning the real sector, our starting point is Naimzada and Pecora (2017), in which a multiplieraccelerator model with a bounded investment function (following Hicks' original intuition) is studied. The goal of Naimzada and Pecora (2017) was to show the possible emergence of instabilities in the dynamics when a nonlinear investment function [The investment function in Naimzada and Pecora (2017) as in the present contribution is shaped according to Hicks' idea of embodying a floor and a ceiling in the evolution of investments, in order to allow the hypothesis of disinvestment and impossibility of an indefinite growth due to obvious resource constraints (see also Puu et al., 2005; Sushko et al., 2010; and Bischi et al. 2001)]. is taken into account and to understand the effect of the introduction of suitable fiscal policy rules. In the present contribution, we extend such a framework considering the interaction with a stock market populated by heterogeneous speculators who place different types of asset demand. In particular, the interaction between real and financial market occurs in both directions. On the financial side of the economy, we assume that the decisions of economic individuals are based on the fundamental value of the financial asset. Such value depends on both an exogenous fundamental value and an endogenous component linked to a suitably weighted current realization of income. On the real side, investments depend, with a certain weight, on the stock market price. In particular, such a weight represents the degree of interaction between the two markets. The resulting setting allows us to understand how the accelerator principle, together with the degree of interaction between the two markets, may influence the dynamics of the economy and the overall business cycle.

We remark that both Naimzada and Pireddu (2015) and Westerhoff (2012) are in analogy with the present one as they both consider the interactions between the two sides of the economy. However, in the present contribution we deal with a completely different setting for the real sector, that is, the multiplier-accelerator. Moreover, in Naimzada and Pireddu (2015) the investment function is linear and it positively depends on the national income, while in Westerhoff (2012) the real market subsystem is described by a stable linear relation, while the financial sector is represented by a cubic one, that is, by a nonlinear relation. The main consequence is that the oscillating behavior can be only ascribed to the financial subsystem. In the present contribution, as in Naimzada and Pecora (2017), oscillations are endogenously generated and stoked by the acceleration principle acting in the real subsystem. This occurs through the nonlinearity of the real subsystem which, in turn, is due to the nonlinearity of the investment function with respect to the change in the level of the national income.

Our research goal is to deepen the understanding of the role of real and financial feedback mechanisms in order to identify, by means of rigorous analytical investigations complemented by numerical simulations, how instability may spread between the integrated markets. To this end, in the present contribution the central parameter is the one that encompasses the degree of interaction. The research is carried on acting at three levels.

First, taking into account two different fiscal policy rules, we investigate their potential to lead the economy toward the steady state national income. We find that a leveladjusting rule with an increasing value of its reactivity with respect to the national income target is able to lead the economy to the desired output level while either level-adjusting or trend-offsetting rules together with suitably integrated markets generate a higher income level with respect to when they are independent. From this viewpoint, the conclusion would be that a suitable high level of market interaction would be beneficial. Such an exclusively static investigation can be misleading, as it is well-known by Westerhoff (2012) that, for example, speculators' decision mechanisms can be the source of complex market dynamics, which foster the emergence of endogenous fluctuations in economic activity that, in turn, affect the assets' fundamental values, with a cascade effect. On the contrary, as in the present setting, it may be the case that the real market, thank to the investment activities, boosts the output and the overall business cycle, which affects the demands placed by speculators in the stock market. In light of this, the government intervention can be seen as an attempt to stabilize business fluctuations.

We then introduce a second level of investigation, in which we analytically study the local stability properties of 
the steady states. We show that the stabilization of the national income may become an intricate issue for the public authority as such policies may also amplify the fluctuations of the business cycle. Such results similar to those of Naimzada and Pecora (2017) extend to the case of interacting markets. In particular, the policy can introduce and/or amplify the fluctuations of the business cycle for any degree of interaction. However, we show that increasing the degree of interaction can have a stabilizing effect in some cases, in particular, for suitable accelerator values and that it never introduces local instability. Hence, also from this perspective, its role is beneficial.

However, also the investigation of local stability is not sufficient to have a complete picture of the possible dynamical behaviors. In fact, with the help of numerical investigations, we analyze the global properties of the model and we find that endogenizing the public expenditure together with the degree of market interaction may imply multistability, that is, the coexistence of different kinds of attractors. In particular, we show that, even if the national income is locally stable independently of the interaction degree, as the interaction degree increases, the stable steady state can coexist with complex attractors, giving rise to erratic trajectories for the national income. From the empirical viewpoint, this level of investigation is essential to reproduce the fluctuations and the recurrent boom-bust sequences observed in the real financial markets together with several stylized facts regarding stock prices and their return (and output as well), such as positive autocorrelation, volatility clustering, and nonnormal distribution characterized by a high kurtosis and fat tails. In the existing literature, the occurrence of complex dynamics able to show qualitative properties of the economic variables was generally linked to the loss of stability of the steady state and ascribable to a local investigation of dynamics. Pushing on the local analysis, the three levels of investigations allow us to conclude that the interaction between the real economy and the stock market may be either beneficial or a trail for the whole economy.

The rest of the paper is organized as follows: Sec. II outlines the features of the two markets; in Sec. III, the analytical results concerning the stability of the steady state equilibrium are presented; Sec. IV presents the numerical simulations; and Sec. V concludes.

\section{THE BASELINE MODEL}

In what follows, we introduce the models we are going to study, in which a real market is integrated with a stock market. The two markets are separately outlined in Subsections II A and II B. The modelling approach we pursue is based, as in Naimzada and Pecora (2017), on a discrete time framework, namely, both the real and the financial markets are studied at time periods $t \in \mathbb{N}$. This also allows us to compare the results to those in the literature, and in particular, to understand the effect of introducing interaction between real and the financial sectors in the economic setting studied in Naimzada and Pecora (2017).

\section{A. The real market}

The benchmark for the real market recalls the seminal business cycle model of Samuelson (see Samuelson, 1939) which incorporates the Keynesian multiplier, a multiplicative factor that relates expenditures to the national income, and the accelerator principle, whereby induced investment is proportional to increases in the national income. In particular, we consider the framework of a closed economy, similar to that studied in Naimzada and Pecora (2017), with the substantial difference that in the present case the possible interaction with a stock market is taken into account.

The macroeconomic equilibrium condition, at any time $t$, is given by

$$
Y_{t}=C_{t}+G_{t}+I_{t}
$$

where $Y_{t}, C_{t}, G_{t}$, and $I_{t}$ represent the national income, the aggregate consumption, the government expenditures, and private investment, respectively. In particular, the setup for the real economy is as follows. Aggregate consumption at time $t$ depends on autonomous consumption $\bar{C}$ and on the previous period national income, that is

$$
C_{t}=\bar{C}+c Y_{t-1}
$$

where $c \in(0,1)$ is an exogenous constant representing the marginal propensity to consume.

Investments are determined by the principle of accelerator. In particular, the investments' function is assumed to be composed of three parts, which are, respectively, an autonomous component and two terms depending on the national income variation and on the financial asset price, and reads as:

$$
I_{t}=\bar{I}+g\left(Y_{t-1}-Y_{t-2}\right)+\omega p P_{t-1} .
$$

The first part $\bar{I}$ represents exogenous investments. The second part $g: \mathbb{R} \rightarrow \mathbb{R}$ is a function which encompasses the component of investments related to the national income variation. In particular, as in Naimzada and Pecora (2017), a possible expression for function $g$ is given by the sigmoid function

$$
g\left(Y_{t-1}-Y_{t-2}\right)=\gamma a_{2}\left(\frac{a_{1}+a_{2}}{a_{1} e^{-\left(Y_{t-1}-Y_{t-2}\right)}+a_{2}}-1\right),
$$

where $\gamma>0$ is the accelerator parameter that tunes the reactivity of investments with respect to the national income variation, $a_{1}, a_{2}$ are positive parameters, representing the lower and upper bounds $\left(-a_{2}\right.$ and $\left.a_{1}\right)$. As a consequence, the component of investments related to the national income variation can increase (respectively decrease) by at most $\gamma a_{1}$ (respectively $\gamma a_{2}$ ) if the national income increases (respectively decreases) from period $t-1$ to $t-2$. This choice is also motivated by the classic literature during the period 1930s-1950s with several papers on macrodynamics such as Kalecki (1935) where a gestation lag for investments is considered, Kaldor (1940) where a nonlinear investment function based on the profit principle is employed, Hicks (1950) who extended the original Samuelson's model with floor and 
ceiling, and Goodwin (1951) who assembled Samuelson's model with a nonlinear delay investment based on the acceleration principle. More recently, the nonlinear accelerator model with investment delays is discussed in Matsumoto (2009) and Matsumoto and Szidarovszky (2015).

The third term $\omega p P_{t-1}$ in (3) links investments' function to the performance of the stock market. In particular, $p$ is a positive parameter, which connects investments to the price of the financial asset, as it is assumed investments' decisions of firms and households depend also on the performance of the stock market, while $\omega \in[0,1]$ measures the degree of interaction between real and stock market. If $\omega=0$, the two markets are independent while if $\omega=1$, the two markets are fully integrated. Moreover, due to the linearity of this last term, we assume that $p$ is suitably small to avoid unhampered increase or decrease in the national income.

Finally, concerning the government expenditures, similar to Naimzada and Pecora (2017), we study two possible fiscal policy rules, namely

- a policy strategy based on a level adjusting rule

$$
G_{t}=\bar{G}+g_{1}\left(Y^{F}-Y_{t-1}\right),
$$

- a policy strategy based on an anti-cyclical rule

$$
G_{t}=\bar{G}+g_{2}\left(Y_{t-2}-Y_{t-1}\right) .
$$

The main difference between (5) and (6) is that according to (5) the expenditures for time $t$ are adjusted proportionally to the difference between the income target $Y^{F}$, which is assumed to be exogenously set by a fiscal authority, and the last national income at time $t-1$, while according to (6) the government adjusts its expenditures proportionally to the deviation of the last observed change in national income level. The latter rule aims at either cooling down or stimulating the economy when there is an upturn or a decline in the output level. Finally, in both (5) and (6), constants $g_{i}$ represent the adjustment speed coefficients (For further details about (5) and (6) we refer to Naimzada and Pecora (2017).

Hence, replacing (2) and (3) [in which we use the expression of $g$ given by (4)] into (1), it follows that the national income can be expressed by

$$
\begin{aligned}
Y_{t}= & \bar{C}+\bar{I}+G_{t}+c Y_{t-1} \\
& +\gamma a_{2}\left(\frac{a_{1}+a_{2}}{a_{1} e^{-\left(Y_{t-1}-Y_{t-2}\right)}+a_{2}}-1\right)+\omega p P_{t-1},
\end{aligned}
$$

where $G_{t}$ is either (5) or (6).

\section{B. Stock market}

We assume that, as in Westerhoff (2012), two types of speculators populate the financial market, namely, fundamentalists and chartists. Moreover, there exists a market maker who sets the price of the financial asset $P_{t}$ by evaluating the market excess demand and determining the price by a linear adjustment mechanism

$$
P_{t}=P_{t-1}+\rho\left(D_{t-1}^{F}+D_{t-1}^{C}\right),
$$

where $\rho>0$ is a positive adjustment parameter, $D_{t-1}^{F}$ and $D_{t-1}^{C}$ are fundamentalists' and chartists' demands, respectively, so that market excess demand is determined by their sum.

Chartists (or technical analysts) make their decision on future assets prices based on price estimation on observing prices time series. They buy stocks if they think price is increasing (bull market) and they sell stocks if they think price is decreasing (bear market). Their demand is defined as

$$
D_{t}^{C}=e\left(P_{t}-F_{t}\right)
$$

where $F_{t}$ is the fundamental value perceived by speculators and $e>0$ is the chartists' reactivity parameter.

On the contrary, fundamentalists base their decision on fundamental values of the market: They invest when stocks are undervalued while they sell when stocks are overvalued, since they believe the stock price will return to its fundamental value. Hence, the order placed by fundamentalists is formalized as

$$
D_{t}^{F}=f\left(F_{t}-P_{t},\right)
$$

where $f>0$ is the fundamentalists' reactivity parameter.

The stock market fundamental value is connected, of course, to the developments of the real economy. The fundamental value of the asset price estimated at time $t$ is described by a weighted average of an exogenous fundamental value $F$ and a component proportional to the national income, that is,

$$
F_{t}=(1-\omega) F+\omega d Y_{t},
$$

where $\omega \in[0,1]$ is the interaction parameter introduced for the investments function in (3) and $d>0$ captures the relation between the fundamental value and the national income [As for $p$ in (3), we assume that $d$ is suitably small to prevent the divergence of fundamental value $F_{t}$. $]$. It is worth noticing that for $\omega \in(0,1]$, the fundamental value of the asset varies over time and if the economy is expanding the national income increases, as well as the fundamental value of the financial asset. Similar to Westerhoff (2012), speculators use the current level of national income as a proxy for the fundamental value. But additionally, the fundamental value is also linked to the real market via the interaction parameter $\omega$. Plugging (11) into (9) and (10), and replacing these into (8), the price equation can be rewritten as

$$
\begin{aligned}
P_{t}= & P_{t-1}+\rho\left(e\left(P_{t-1}-(1-\omega) F-\omega d Y_{t-1}\right)\right. \\
& \left.+f\left((1-\omega) F+\omega d Y_{t-1}-P_{t-1}\right)\right) .
\end{aligned}
$$

We notice that it is common to assume (see Naimzada and Pireddu, 2015) that, since the speed of price adjustment in a financial market is much larger than that of good market prices, the speed of adjustment in the stock market is infinite, namely, $\rho \rightarrow \infty$. In such way, we obtain the equilibrium condition in that market as

$$
\begin{aligned}
\frac{P_{t}-P_{t-1}}{\rho}= & e\left(P_{t-1}-(1-\omega) F-\omega d Y_{t-1}\right) \\
& +f\left((1-\omega) F+\omega d Y_{t-1}-P_{t-1}\right)
\end{aligned}
$$

and when $\rho \rightarrow \infty$ we get 


$$
\begin{gathered}
e\left(P_{t-1}-(1-\omega) F-\omega d Y_{t-1}\right)+f((1-\omega) F \\
\left.+\omega d Y_{t-1}-P_{t-1}\right)=D_{t-1}^{F}+D_{t-1}^{C}=0,
\end{gathered}
$$

namely, depending on the national income $Y_{t}$, the price $P_{t}$ is such that the aggregated market demand provides a null excess demand. Indeed, from the previous equation we obtain (As in Naimzada and Pireddu (2014), we assume that fundamentalists and chartists have different reactivities, i.e., $e \neq f$, as, otherwise, the dynamic equation (12) of the stock market would reduce to $P_{t}=P_{t-1}$.)

$$
P_{t}=(1-\omega) F+\omega d Y_{t},
$$

which means that price is given by a weighted average of the exogenous fundamental value and of a component proportional to the national income.
In order to study the interaction between the two markets, it is convenient to replace the investment function (13) into the national income relationship (7), obtaining

$$
\begin{aligned}
Y_{t}= & \bar{C}+\bar{I}+G_{t}+\omega p(1-\omega) F+\left(c+\omega^{2} p d\right) Y_{t-1} \\
& +\gamma a_{2}\left(\frac{a_{1}+a_{2}}{a_{1} e^{-\left(Y_{t-1}-Y_{t-2}\right)}+a_{2}}-1\right),
\end{aligned}
$$

which is a second order difference equation, which can be reduced to a system of two difference equations by introducing the new variable $Z_{t-1}=Y_{t-2}$. Finally, replacing $G_{t}$ with either (5) or (6) and setting $A \equiv \bar{C}+\bar{G}+\bar{I}$ to collect the autonomous components, we can introduce functions $T_{i}: \mathbb{R}_{+}^{2} \rightarrow \mathbb{R}^{2}$, for $i=1,2$, respectively defined by

$$
T_{1}:\left\{\begin{array}{l}
Y_{t}=A+Y^{F} g_{1}+\left(d p \omega^{2}+c-g_{1}\right) Y_{t-1}+p \omega(1-\omega) F+\gamma a_{2}\left(\frac{a_{1}+a_{2}}{a_{2}+a_{1} e^{Z_{t-1}-Y_{t-1}}}-1\right) \\
Z_{t}=Y_{t-1}
\end{array}\right.
$$

representing the model in which the level adjusting rule is adopted and

$$
T_{2}:\left\{\begin{array}{l}
Y_{t}=A+g_{2} Z_{t-1}+\left(d p \omega^{2}+c-g_{2}\right) Y_{t-1}+p \omega(1-\omega) F+\gamma a_{2}\left(\frac{a_{1}+a_{2}}{a_{2}+a_{1} e^{Z_{t-1}-Y_{t-1}}}-1\right) \\
Z_{t}=Y_{t-1}
\end{array}\right.
$$

representing the model in which the anti-cyclical rule is adopted.

\section{ANALYTICAL RESULTS ON LOCAL STABILITY}

In this section, we investigate models (14) and (15), studying the local stability of the steady states. In this respect, we are going to pay specific attention on the role of the interaction parameter $\omega$, of the accelerator $\gamma$ and of the reactivity of the fiscal policies $g_{i}$.

Before presenting the results, it is worth noticing that if we consider $\omega=0$, that is, the case of independent real and financial markets, we reduce to the setting considered in Naimzada and Pecora (2017). In particular, we recall from Naimzada and Pecora (2017) that if we also set $g_{i}=0$, the unique steady state $Y^{s}=A /(1-c)$ coincides with the equilibrium of the original Samuelson multiplier-accelerator model (see Samuelson, 1939). We report the following Proposition from Naimzada and Pecora (2017).

Proposition 1. The Samuelson equilibrium $Y^{s}$ is locally stable provided that

$$
\gamma<\gamma^{N S}=\frac{a_{1}+a_{2}}{a_{1} a_{2}}
$$

Moreover, at $\gamma=\gamma^{N S}$ a supercritical Neimark-Sacker bifurcation occurs. [The reader may refer to Naimzada and Pecora (2017) for the proof.]
Furthermore, if we consider the stock market to be independent on the real market, the asset price equilibrium value is $F$ and it is always stable because of the equilibrium hypothesis.

\section{A. Model with a level-adjusting fiscal policy rule}

When considering a level-adjusting rule, such as in (5), we refer to a government who decides to compensate for the difference between the last output realization and a benchmark value (e.g., a long run average value or a value near the full employment level). Hence, the dynamics of the model is also dependent on how the fiscal authority aims at pushing the national income to the benchmark output level. So does the steady state.

A straightforward computation shows that the unique steady state equilibrium of the map $T_{1}$ is

$$
Y^{* *}=Z^{* *}=\frac{A+Y^{F} g_{1}+p \omega(1-\omega) F}{1+g_{1}-c-d p \omega^{2}},
$$

which is strictly positive and well defined for any interaction degree $\omega \in[0,1]$ provided that

$$
1+g_{1}-c-p d>0 .
$$

We stress that condition (16) requires that parameters $p$ and $d$, which respectively connect investments to the price of the financial asset and the fundamental value to the national 
income, are suitably small. Such constant is in agreement with the economic considerations about $p$ and $d$ of Sec. II. If $\omega=0$, we indeed retrieve the steady state found in Naimzada and Pecora (2017), Sec. III A. Moreover, we remark that $Y^{* *}$ is increasing with respect to $F$ and $Y^{F}$, while concerning the role of $g_{1}$ and $\omega$ we have the following:

Proposition 2. The national income equilibrium $\left(Y^{* *}, Z^{* *}\right)$ converges toward $Y^{F}$ as $g_{1} \rightarrow+\infty$. The convergence is monotonically increasing if

$$
Y^{F}>\frac{A+p \omega(1-\omega) F}{1-c-d p \omega^{2}}=Y^{*},
$$

while it is monotonically decreasing if $Y^{F}<Y^{*}$.

Let

$$
\frac{d\left(A+Y^{F} g_{1}\right)}{1+g_{1}-c}<F<\frac{2 d\left(A+Y^{F} g_{1}\right)}{1+g_{1}-c-d p} .
$$

Then, there exists $\tilde{\omega} \in(0,1)$ so that the national income equilibrium $Y^{* *}$ is strictly increasing on $[0, \tilde{\omega})$ and strictly decreasing on $(\tilde{\omega}, 1]$, reaching its maximum value for $\tilde{\omega}$.

Conversely, if (18) does not hold, the national income $Y^{* *}$ equilibrium is strictly increasing on $\omega \in[0,1]$ and attains its maximum value $\left(A+Y^{F} g_{1}\right) /\left(1+g_{1}-c-d p\right)$ at $\omega=1$.

In both cases, the national income when the two markets are fully integrated is always greater than when they are independent.

Proof. The first assertion is straightforward. The behavior with respect to $g_{1}$ is obtained noticing that

$$
\frac{\partial Y^{* *}}{\partial g}=\frac{-F p \omega(1-\omega)-A+Y^{F}\left(1-c-d p \omega^{2}\right)}{\left(d p \omega^{2}+c-g_{1}-1\right)^{2}} .
$$

Concerning $\omega$, a simple computation shows that

$$
\frac{\partial Y^{* *}}{\partial \omega}=\frac{F p\left(g_{1}-c+1\right)+2 p \omega\left(A d-F+F c-F g+Y^{F} d g_{1}\right)+F d p^{2} \omega^{2}}{\left(1+g_{1}-c-d p \omega^{2}\right)^{2}}
$$

whose sign is determined by the sign of the numerator. Let us introduce the function $q:[0,1] \rightarrow \mathbb{R}$, where $q(\omega)$ is the numerator of $\partial Y^{* *} / \partial \omega$. Function $q$ represents a convex parabola with $q(0)=F p\left(g_{1}-c+1\right)>0$ and for which $q(1)=2 d p\left(A+Y^{F} g_{1}\right)-p F\left(g_{1}-c+1-d p\right)$ and $q^{\prime}(1)$ $=2 d p\left(A+Y^{F} g_{1}\right)-2 p F\left(g_{1}-c+1-d p\right)$. As a consequence, we have

$$
q(1)>0 \Longleftrightarrow F<\frac{2 d\left(A+Y^{F} g_{1}\right)}{g_{1}-c+1-d p}=2 \tilde{F}
$$

and

$$
q^{\prime}(1)>0 \Longleftrightarrow F<\frac{d\left(A+Y^{F} g_{1}\right)}{g_{1}-c+1-d p}=\tilde{F} .
$$

If $q(1)<0$ (which implies $q^{\prime}(1)<0$ ) since $q$ is convex and $q(0)>0$, we have that there exists $\tilde{\omega}$ such that $q(\omega)>0$ for $\omega \in[0, \tilde{\omega})$ and $q(\omega)<0$ for $\omega \in(\tilde{\omega}, 1]$ so that $\partial Y^{* *} / \partial \omega$ $>0$ for $\omega \in[0, \tilde{\omega})$ and $\partial Y^{* *} / \partial \omega<0$ for $\omega \in(\tilde{\omega}, 1]$, which means that $Y^{* *}$ is increasing for small values of $\omega$ and decreasing for large values of $\omega$.

If $q(1) \geq 0$ and $q^{\prime}(1) \leq 0$, since $q$ is convex and $q(0)$ $>0$ we have that $q(\omega) \geq 0$ for any $\omega \in[0,1]$, so $\partial Y^{* *} /$ $\partial \omega \geq 0$ for any $\omega \in[0,1]$ and $Y^{* *}$ is strictly increasing.

Conversely, if $q(1)>0$ and $q^{\prime}(1) \geq 0$, in principle we may have several situations. Let $\omega_{V}$ be the vertex of $q$, for which we indeed have $\omega_{V}<1$. In this case, we have $q(\omega)$ $>0$ for any $\omega \in[0,1]$ if and only if $\omega_{V} \leq 0$ (in which case $q$ is strictly increasing) or $q\left(\omega_{V}\right)>0$ and $\omega_{V}>0$.

We have $\omega_{V}=\left(F\left(1+g_{1}-c\right)-d\left(A+Y^{F} g_{1}\right)\right) /(F d p)$, which is strictly positive provided that

$$
F\left(1+g_{1}-c\right)-d\left(A+Y^{F} g_{1}\right)>0,
$$

and we have

$$
q\left(\omega_{V}\right)=\frac{-F^{2}\left(g_{1}-c+1\right)\left(1+g_{1}-c-d p\right)+2 F d\left(A+Y^{F} g_{1}\right)\left(g_{1}-c+1\right)-d^{2}\left(A+Y^{F} g_{1}\right)^{2}}{F d}
$$

We notice that

$$
\begin{aligned}
-F^{2} & \left(g_{1}-c+1\right)\left(1+g_{1}-c-d p\right) \\
& +F d\left(A+Y^{F} g_{1}\right)\left(g_{1}-c+1\right) \\
= & F\left(g_{1}-c+1\right)\left[-F\left(1+g_{1}-c-d p\right)\right. \\
& \left.+d\left(A+Y^{F} g_{1}\right)\right] \geq 0
\end{aligned}
$$

since $q^{\prime}(1) \geq 0$, and that

$$
\begin{aligned}
& F d\left(A+Y^{F} g_{1}\right)\left(g_{1}-c+1\right)-d^{2}\left(A+Y^{F} g_{1}\right)^{2} \\
& \quad=d\left(A+Y^{F} g_{1}\right)\left[F\left(g_{1}-c+1\right)-d\left(A+Y^{F} g_{1}\right)\right]>0
\end{aligned}
$$

thanks to (19). This implies that $q\left(\omega_{V}\right)>0$ and hence $q(\omega)>0$ for any $\omega \in[0,1]$ so that $\partial Y^{* *} / \partial \omega \geq 0$ for any $\omega \in[0,1]$ and $Y^{* *}$ is strictly increasing.

To prove the last assertion, it is sufficient to note that the numerator of $Y^{* *}$ is equal to $A+Y^{F} g_{1}$ for both $\omega=0$ and 
$\omega=1$, while for $\omega=0$ its denominator is $1+g_{1}-c$ $>1+g_{1}-c-d p$, which is the denominator for $\omega=1$. This concludes the proof.

We notice that the value $Y^{*}$, which has been introduced in the previous Proposition, is actually the steady state of the model in which no policy rule (i.e., $g_{1}=0$ ) is adopted and the government expenditure is constant $\bar{G}$.

To study the local stability of $\left(Y^{* *}, Z^{* *}\right)$, we introduce the threshold values

$$
\begin{aligned}
& \gamma_{1}^{f}=\frac{a_{1}+a_{2}}{2 a_{1} a_{2}}\left(-1-c+g_{1}-d p \omega^{2}\right), \quad \gamma_{1}^{n s}=\frac{a_{1}+a_{2}}{a_{1} a_{2}}, \\
& \omega_{1}^{f}=\sqrt{\frac{1}{d p}\left(g_{1}-c-\frac{2 a_{1} a_{2} \gamma}{a_{1}+a_{2}}-1\right)}, \\
& g_{1}^{f}=c+d p \omega^{2}+\frac{2 a_{1} a_{2} \gamma}{a_{1}+a_{2}}+1,
\end{aligned}
$$

where $\omega_{1}^{f}$ is defined provided that $g_{1}-c-1-2 a_{1} a_{2} \gamma /$ $\left(a_{1}+a_{2}\right) \geq 0$

In the next Proposition, we investigate the local stability of $\left(Y^{* *}, Z^{* *}\right)$ showing the only possible behaviors on varying parameters $\omega, \gamma$ and $g_{1}$. We stress that an unconditionally stable/unstable scenario is realized when the steady state is locally asymptotically stable/unstable independently of the parameter values; a stabilizing/destabilizing scenario occurs when the steady state is locally asymptotically stable only below/above a given threshold and unstable otherwise; a mixed scenario arises when the steady state is locally asymptotically stable only for intermediate parameter values, between two stability thresholds, and unstable otherwise.

Proposition 3. The only possible scenarios on increasing the degree of interaction $\omega$ are the unconditionally unstable, the unconditionally stable, and the stabilizing scenario. In this last case, a period halving bifurcation occurs at $\omega=\omega_{1}^{f}$.

The only possible scenarios on increasing the adjustment speed $g_{1}$ are the unconditionally unstable and the destabilizing scenario. In this last case, a flip bifurcation occurs at $g=g_{1}^{f}$.

The only possible scenarios on increasing the accelerator $\gamma$ are the unconditionally unstable scenario, a destabilizing scenario, in which a Neimark-Sacker bifurcation occurs at $\gamma=\gamma_{1}^{n s}$, and a mixed scenario in which a period halving bifurcation occurs at $\gamma=\gamma_{1}^{f}$ and a Neimark-Sacker bifurcation occurs at $\gamma=\gamma_{1}^{n s}$, being $\left(Y^{* *}, Z^{* *}\right)$ stable for $\gamma \in\left(\gamma_{1}^{f}, \gamma_{1}^{n s}\right)$.

Proof. The Jacobian matrix of the map (14) is

$$
J_{1}=\left(\begin{array}{cc}
c-g_{1}+d p \omega^{2}+\gamma \frac{a_{1} a_{2} e^{Z_{t-1}-Y_{t-1}}\left(a_{1}+a_{2}\right)}{\left(a_{2}+a_{1} e^{Z_{t-1}-Y_{t-1}}\right)^{2}} & -\gamma \frac{a_{1} a_{2} e^{Z_{t-1}-Y_{t-1}}\left(a_{1}+a_{2}\right)}{\left(a_{2}+a_{1} e^{Z_{t-1}-Y_{t-1}}\right)^{2}} \\
1 & 0
\end{array}\right),
$$

which evaluated at the steady state provides

$$
J_{1}^{* *}=\left(\begin{array}{cc}
c-g_{1}+d p \omega^{2}+\gamma \frac{a_{1} a_{2}}{a_{1}+a_{2}} & -\gamma \frac{a_{1} a_{2}}{a_{1}+a_{2}} \\
1 & 0
\end{array}\right),
$$

where $\operatorname{Tr}\left(J_{1}^{* *}\right)=c-g_{1}+d p \omega^{2}+\gamma a_{1} a_{2} /\left(a_{1}+a_{2}\right)$ and $\operatorname{det}\left(J_{1}^{* *}\right)$ $=\gamma a_{1} a_{2} /\left(a_{1}+a_{2}\right)$ are, respectively, the trace and the determinant of matrix $J_{1}^{* *}$.

According to the usual Jury's conditions (see, e.g., Medio and Lines, 2005), the steady state is locally asymptotically stable provided that

$$
\begin{aligned}
1-\operatorname{Tr}\left(J_{1}^{* *}\right)+\operatorname{det}\left(J_{1}^{* *}\right) & =1+g_{1}-c-d p \omega^{2}>0, \\
1-\operatorname{det}\left(J_{1}^{* *}\right) & =\frac{a_{1}+a_{2}-a_{1} a_{2} \gamma}{a_{1}+a_{2}}>0, \\
1-\operatorname{Tr}\left(J_{1}^{* *}\right)+\operatorname{det}\left(J_{1}^{* *}\right) & =c-g_{1}+d p \omega^{2}+\frac{2 a_{1} a_{2} \gamma}{a_{1}+a_{2}}+1>0 .
\end{aligned}
$$

The first condition is always satisfied thanks to assumption (16), while the second one requires $\gamma<\gamma_{1}^{n s}$. Solving the last stability condition with respect to $\gamma$ we find $\gamma>\gamma_{1}^{f}$, so inequalities (21b) and (21c) are simultaneously fulfilled provided that $\gamma_{1}^{f}<\gamma<\gamma_{1}^{n s}$. Since we need $\gamma>0$, this means that if $\gamma_{1}^{f} \geq \gamma_{1}^{n s}$ we have the unconditionally unstable scenario, if $\gamma_{1} \leq 0$ we have the destabilizing scenario, and if $0<\gamma_{1}^{f}<\gamma_{1}^{n s}$ we have a mixed scenario.

If we consider (21) both with respect to $\omega$ and $g$, if $\gamma$ $\geq \gamma_{1}^{n s}$, condition (21b) is not fulfilled independently of $\omega$ and $g$, so we have the unconditionally unstable scenarios with respect to both $\omega$ and $g$. Now, we assume $\gamma<\gamma_{1}^{n s}$ and we solve (21c) with respect to $\omega$. Thanks to the positivity of the coefficient of $\omega^{2}$, condition (21c) is either satisfied for any $\omega \in \mathbb{R}$ or for $|\omega|>\omega_{1}^{f}$. Since we need $\omega \in[0,1]$, in the former case or in the latter one for $\omega_{1}^{f}>1$, the steady state is unconditionally stable, while in the latter case for $\omega_{1}^{f} \geq 1$ we have a stabilizing scenario.

Finally, assuming $\gamma<\gamma_{1}^{n s}$ and solving (21c) with respect to $g_{1}$ we find $g_{1}<g_{1}^{f}$, so we are in the destabilizing scenario. Recalling that when $1-\operatorname{det}\left(J_{1}^{* *}\right)=0$ we have a NeimarkSacker bifurcation while a flip bifurcation occurs when $1-\operatorname{Tr}\left(J_{1}^{* *}\right)+\operatorname{det}\left(J_{1}^{* *}\right)=0$ allows identifying the kind of bifurcations occurring at $\gamma_{1}^{f}, \gamma_{1}^{n s}, \omega_{1}^{f}$ and $g_{1}^{f}$. This concludes the proof.

A graphical illustration of the stability regions in the parameter planes $(\gamma, \omega),\left(\gamma, g_{1}\right)$ and $\left(g_{1}, \omega\right)$ is reported in Fig. 1, for which we set $A=50, Y^{F}=1, c=0.8, d=0.7$, $p=1, F=50, a_{1}=3$, and $a_{2}=0.2$. From Fig. 1 it is evident that all the possible scenarios described by Proposition 3 actually occur for some parameter configurations. As we 


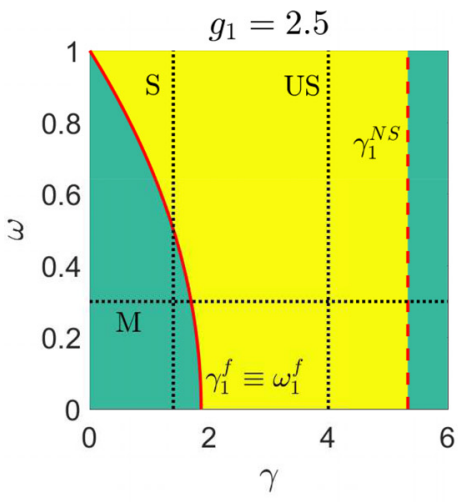

(a)

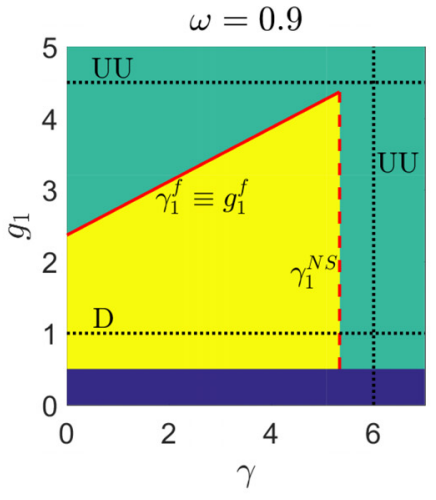

(b)

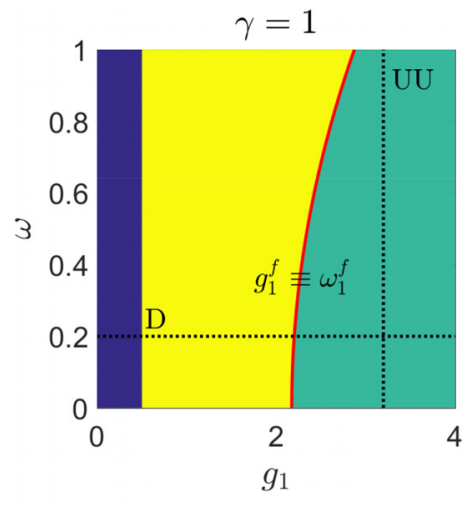

(c)

FIG. 1. Stability regions in $(\gamma, \omega),\left(\gamma, g_{1}\right)$, and $\left(g_{1}, \omega\right)$ parameter planes. Yellow, green, and blue colors, respectively, identify regions in which the steady state is stable, unstable, and does not fulfill the assumption (16). Solid and dashed red lines, respectively, represent flip and Neimark-Sacker bifurcation curves. Black dotted lines point out unconditionally unstable (UU), unconditionally stable (US), destabilizing (D), stabilizing (S), and mixed (M) scenarios.

can see from Fig. 1(a), for a fixed value of the accelerator parameter, the stability region (represented in yellow) of $\left(Y^{* *}, Z^{* *}\right)$ enlarges as the interaction degree increases. Similarly, for a fixed policy adjustment speed, increasing $\omega$ has a stabilizing effect [Fig. 1(c)].

Moreover, in agreement with the results of Proposition 3 , we may have both a single and a double stability threshold with respect to the accelerator parameter $\gamma$ [Figs. 1(a) and 1(b)]. We notice that, as in Naimzada and Pecora (2017), an increasing reactivity of the public expenditures with respect to the deviations of the national income to its target may reduce the size of the stability region. Finally, looking at Fig. 1 (c), we can confirm that the steady state is locally stable when the public intervention is not extreme as well as the degree of interaction between the real and the financial sector is sufficiently large.

The previous results regarding the stability of the steady state equilibrium with respect to the interaction degree $\omega$ and the reactivity of the fiscal policy $g_{1}$ can be related to the by now accepted wave of globalization and the recent global financial crisis. While the origin of the crisis is attributed to the financial sectors, there are also some counterparts in the real economy. Fiscal policy is then called upon to provide additional stabilization in a scenario in which the integration between the two sides of the economy is becoming more tightened.

In Sec. IV, we will deepen the investigation about the dynamics arising when the steady state loses stability through numerical simulations. We stress that Proposition 3 shows that, as a consequence of either a flip or a NeimarkSacker bifurcation, both periodic and quasi-periodic trajectories are possible. The relevance of a Neimark-Sacker bifurcation is connected to its capability to explain the emergence of endogenous quasi-periodic phenomena, resembling the alternation of periods of high and low economic activity (business cycles), without requiring to introduce into the model exogenous ad-hoc shock components. Similarly, a cascade of period-doublings can lead to the emergence of chaotic dynamics, resembling indeterministic trajectories. For further discussions about the economic interpretation of different dynamics, we refer to Hommes (2013).

\section{B. Model with an anti-cyclical rule}

In this part, we assume that the fiscal authority aims at offsetting the national income trends. Policy makers increase (decrease) the government expenditures when the national income has just been falling (rising) with the aim of smoothing out the business cycle. Especially after the recent financial crisis, evidence showed that fiscal policy may be an appropriate countercyclical tool when the financial sector is weak or the output gap is particularly large. Keeping this in mind, the model we are going to analyze is described by map $T_{2}$ in (15).

Simple computations allows us to determine the unique steady state of the system (15), which is given by

$$
Y^{* * *}=Z^{* * *}=\frac{A+p \omega(1-\omega) F}{1-c-d p \omega^{2}},
$$

on which we make the assumption (Notice that this is the same condition as in the model without government expenditures.)

$$
1-c-d p>0
$$

to guarantee the economic meaningfulness of $Y^{* * *}$ for any interaction degree. Similar to condition (16), such condition is fulfilled when parameters $p$ and $d$ are suitably small. If $\omega=0$, we again retrieve the steady state found in Naimzada and Pecora (2017), Sec. III B, which coincides with the Samuelson equilibrium $Y^{S}$. We remark that $Y^{* * *}$ does not depend on the speed of adjustment $g_{2}$ of the policy rule, while it is increasing with respect to $F$ and $Y^{F}$. On varying the degree of interaction, we obtain the following Proposition.

Proposition 4. Let

$$
\frac{d A}{1-c}<F<\frac{2 d A}{1-c-d p}
$$

Then, there exists $\tilde{\omega} \in(0,1)$ so that the national income $Y^{* * *}$ is strictly increasing on $[0, \tilde{\omega})$ and strictly decreasing on $(\tilde{\omega}, 1]$, reaching its maximum value for $\tilde{\omega}$.

Conversely, if (22) is not fulfilled, the national income $Y^{* * *}$ is strictly increasing on $\omega \in[0,1]$ and attains its maximum value $A /(1-c-d p)$ at $\omega=1$. 
In both cases, the national income when the two markets are fully integrated is always greater than when they are independent.

Proof. Comparing $Y^{* * *}$ with $Y^{* *}$, it is evident that the former steady state is obtained by formally setting $g_{1}=0$ in the latter one. Proposition 4 can be then proved following step by step the proof of Proposition 2.

The only possible scenarios arising with respect to the local stability of $\left(Y^{* * *}, Z^{* * *}\right)$ are investigated in Proposition 5 , in which we use the values

$$
\begin{aligned}
& \gamma_{2}^{f}=\frac{a_{1}+a_{2}}{2 a_{1} a_{2}}\left(-1-c+2 g_{2}-d p \omega^{2}\right), \quad \gamma_{2}^{n s}=\frac{a_{1}+a_{2}}{a_{1} a_{2}}, \\
& \omega_{2}^{f}=\sqrt{\frac{1}{d p}\left(g_{2}-c-\frac{2 a_{1} a_{2} \gamma}{a_{1}+a_{2}}-1\right)}, \\
& g_{2}^{f}=c+d p \omega^{2}+\frac{2 a_{1} a_{2} \gamma}{a_{1}+a_{2}}+1,
\end{aligned}
$$

where $\omega_{2}^{f}$ is defined provided that $g_{2}-c-1-2 a_{1} a_{2} \gamma /\left(a_{1}\right.$ $\left.+a_{2}\right) \geq 0$. The following Proposition summarizes the results on local stability of the $\left(Y^{* * *}, Z^{* * *}\right)$ steady state.

Proposition 5. The only possible scenarios on increasing the degree of interaction $\omega$ are the unconditionally unstable, the unconditionally stable, and the stabilizing scenario. In this last case, a period halving bifurcation occurs at $\omega=\omega_{2}^{f}$.

The only possible scenarios on increasing the adjustment speed $g_{2}$ are the unconditionally unstable and the destabilizing scenario. In this last case, a flip bifurcation occurs at $g=g_{2}^{f}$.

The only possible scenarios on increasing the accelerator $\gamma$ are the unconditionally unstable scenario, a destabilizing scenario, in which a Neimark-Sacker bifurcation occurs at $\gamma=\gamma_{2}^{n s}$, and a mixed scenario in which a period halving bifurcation occurs at $\gamma=\gamma_{2}^{f}$ and a Neimark-Sacker bifurcation occurs at $\gamma=\gamma_{2}^{n s}$, being $\left(Y^{* * *}, Z^{* * *}\right)$ stable for intermediate values of $\gamma \in\left(\gamma_{2}^{f}, \gamma_{2}^{n s}\right)$

Proof. Proceeding as in the proof of Proposition 3, we compute the Jacobian matrix of (15)

$$
J_{2}=\left(\begin{array}{cc}
c-g_{2}+d p \omega^{2}+\gamma \frac{a_{1} a_{2} e^{\left(Z_{t-1}-Y_{t-1}\right)}\left(a_{1}+a_{2}\right)}{\left(a_{2}+a_{1} e^{\left(Z_{t-1}-Y_{t-1}\right)}\right)^{2}} & g_{2}-\gamma \frac{a_{1} a_{2} e^{\left(Z_{t-1}-Y_{t-1}\right)}\left(a_{1}+a_{2}\right)}{\left(a_{2}+a_{1} e^{\left(Z_{t-1}-Y_{t-1}\right)}\right)^{2}} \\
1 & 0
\end{array}\right)
$$

and we evaluate it at the steady state, obtaining

$$
J_{2}^{* * *}=\left(\begin{array}{cc}
c-g_{2}+d p \omega^{2}+\gamma \frac{a_{1} a_{2}}{a_{1}+a_{2}} & g_{2}-\gamma \frac{a_{1} a_{2}}{a_{1}+a_{2}} \\
1 & 0
\end{array}\right)
$$

where $\operatorname{Tr}\left(J_{2}^{* * *}\right)=c-g_{2}+d p \omega^{2}+\gamma\left(a_{1} a_{2}\right) /\left(a_{1}+a_{2}\right)$ and $\operatorname{det}\left(J_{2}^{* * *}\right)=\gamma\left(a_{1} a_{2}\right) /\left(a_{1}+a_{2}\right)-g_{2}$ are, respectively, the trace and the determinant of $J^{* * *}$. Jury's conditions guarantee the local asymptotic stability of the steady state when

$$
\left\{\begin{array}{l}
1-c-d p \omega^{2}>0 \\
a_{1}+a_{2}-a_{1} a_{2} \gamma>0 \\
c-2 g_{2}+d p \omega^{2}+\frac{2 a_{1} a_{2} \gamma}{a_{1}+a_{2}}+1>0 .
\end{array}\right.
$$

Conditions (24) are obtained by setting $g_{1}=2 g_{2}$ in the stability conditions (21) referred to the model with the level adjusting policy rule. This means that thresholds (23) are obtained by replacing $g_{1}$ with $2 g_{2}$ in (20) and that the resulting stability regions of $\left(Y^{* * *}, Y^{* * *}\right)$ for (15) are in one-to-one correspondence with those of $\left(Y^{* *}, Y^{* *}\right)$ for (14). This concludes the proof.

The possible stability scenarios under the anti-cyclical rule are the same as those under the level adjusting rule. Moreover, we have $\gamma_{1}^{n s}=\gamma_{2}^{n s}$ and $g_{1}^{f}=g_{2}^{f}$, while, if we compare the policy rules for the same speed of adjustments $g_{1}=g_{2}$, we have $\gamma_{1}^{f}<\gamma_{2}^{f}$ and $\omega_{1}^{f}<\omega_{2}^{f}$. This result, recalling Propositions 3 and 5, allows us to concluding that the stability region of $\left(Y^{* *}, Y^{* *}\right)$ for (14) always includes that of
$\left(Y^{* * *}, Y^{* * *}\right)$ for $(15)$. All the considerations we made at the end of Sec. III A about the role of parameters, in particular on the degree of interaction, still hold. Similarly, stability regions reported in Fig. 1 are qualitatively the same of those for the model considered in the present section (Recalling the proof of Proposition 5, stability regions can be simply obtained applying transformation $g_{1}=2 g_{2}$.)

\section{NUMERICAL SIMULATIONS}

In this section, we complement the analytical study of local stability performed in Sec. III with several numerical investigations. The goal is to study the possible dynamics when trajectories do not converge toward the steady state and to check whether the nonlinear, possibly complex, dynamical behavior of economic variables fosters the emergence of stylized facts in the time series of prices. In particular, we are interested in the effects, on the dynamics of the national income $Y_{t}$, of the interaction degree between real and financial markets. We stress that we just present results for the model with the level adjusting policy rule, since we extensively checked through simulations that the results are qualitatively the same when the anti-cyclical rule is considered. So, all the comments and conclusions of this section can be extended to model (15), too. All the results reported in this section are obtained for the same parameter setting used for the stability regions of Fig. 1, namely, for $A$ $=50, Y^{F}=1, c=0.8, d=0.7, p=1, F=50, a_{1}=3$, and $a_{2}=0.2$, while $\omega, g_{1}$ and $\gamma$ are specified time by time. 
First, we investigate what happens when the steady state loses stability, reporting in Figs. 2(a)-2(c) three black bifurcation diagrams obtained on varying $\omega, \gamma$, and $g_{1}$, setting initial conditions $\left(Y_{0}, Z_{0}\right)$ suitably close to the steady state. The bifurcation diagram reported in Fig. 2(a) is obtained setting $\gamma=1.4$ and $g_{1}=2.5$ and confirms the stabilizing role of the interaction degree. In agreement with (20), when $\omega$ decreases below $\omega_{1}^{f}=0.5$, the steady state loses stability through a period-doubling bifurcation, giving rise to a period- 2 cycle, which remains stable for any $\omega<\omega_{1}^{f}$. Period-doubling bifurcations, as the policy reactivity $g_{1}$ increases [Fig. 2(b)] and as the accelerator $\gamma$ decreases [Fig. 2(c)], provide qualitatively similar stable period-2 cycles. We remark that such behavior is robust, and in all the simulations we performed we observed that period- 2 cycles arising from flip bifurcations of the steady state remains locally stable on decreasing (for $\omega$ and $\gamma$ ) or increasing (for $g_{1}$ ) the bifurcation parameter. We notice that, recalling (20), $g_{1}^{f}$ $\approx 2.203$, obtained for $\gamma=1$ and $\omega=0.2$, and $\gamma_{1}^{f} \approx 1.6987$, obtained for $g_{1}=2.5$ and $\omega=0.3$, are in agreement with the flip threshold we find in Figs. 2(b) and 2(c).

Conversely, if we set $g_{1}=2.5$ and $\omega=0.3$, as the accelerator increases above $\gamma_{1}^{n s} \approx 5.333$, a Neimark-Sacker bifurcation occurs and $Y_{t}$ follows quasi-periodic trajectories [Fig. 2(c)]. In many business cycle models, the underlying economic variables usually tend to converge to a steady state (made up of, e.g., a constant output level, a constant stock of capital). Usually business cycles are seen as emerging from shocks that perturb an otherwise stable environment. But this is not always the case, as economic forces may naturally
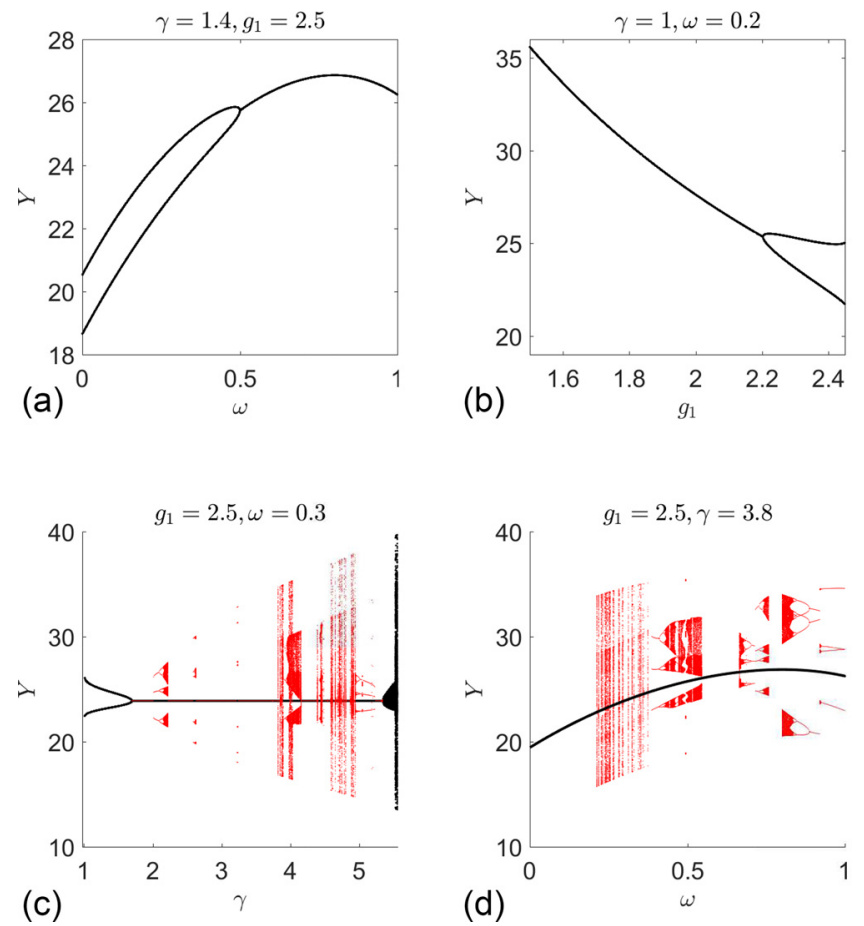

FIG. 2. Bifurcation diagrams with respect to $\omega, \gamma$, and $g_{1}$. Black bifurcation diagrams are obtained with an initial datum which is suitably close to the steady state. The red bifurcation diagrams reported in (c) and (d) show multistability, with coexisting attractors and complex dynamics, that can occur even when the steady state is unconditionally stable, as in (d). produce cyclical phenomena. That is, in the absence of any shocks, economic forces by themselves may favor recurrent periods of high economic activity followed by periods of low economic activity. This outcome emerges, for example, if the underlying system generates a closed invariant curve. When a supercritical Neimark-Sacker bifurcation occurs, indefinitely repeating fluctuations take place. More precisely, in economics, this kind of bifurcation is associated with endogenous oscillations or self-sustaining cycles, to refer to the business cycle arising from endogenous forces that foster the stimulus of economic and financial activity. Such stimulus, in turn, causes the economy to be naturally unstable so that it would undergo boom and bust dynamics even in the absence of any stochastic disturbances (see, e.g., the works of Kaldor, 1940; Goodwin, 1951; and Bischi et al., 2001 for the analysis of business cycle models and the related bifurcations arising in their settings).

However, the local stability analysis and the dynamics arising from the destabilization of the steady state just provide a partial picture of the overall possible phenomena. Figures 2(c) and 2(d) show that coexistence among different attractors is possible. The red bifurcation diagrams superimposed to the black ones are obtained by considering $500^{2}$ possible initial data uniformly distributed on the square [15, $37]^{2}$ [Fig. 2(c)] and on the square $[10,40]^{2}$ [Fig. 2(d)], and representing only those points belonging to trajectories that do not correspond to the stable steady state or to the period2 cycle and the quasi-periodic trajectories arising from the destabilization of $\left(Y^{* *}, Y^{* *}\right)$. As we can see, the resulting structure is quite complex, and the numerical investigations suggest that coexisting attractors can appear and disappear on varying both $\gamma$ and $\omega$. In particular, we have that the stable steady state can coexist with complex, possibly chaotic attractors, even when $\left(Y^{* *}, Y^{* *}\right)$ is unconditionally stable, as, for example, in the case reported in Fig. 2(d).

Such results point out that it may be very difficult that trajectories converge toward the steady state, even when it is locally stable, even more so under the effect of exogenous indeterministic fluctuations. To investigate more in depth such phenomena, we focus on some representative situations arising in the simulations reported in Fig. 2. The corresponding basins of attraction are collected in Figs. 3(a)-3(f), which exhibits the coexistence between the stable steady state with periodic or complex attractors. When different attractors coexist in the phase-space, the structure of their basins of attraction, and the dependence of the basin boundaries to the parameters of the model, plays a crucial role in the long-run behavior of the economy under scrutiny. Eventualities of attractors coexistence have been reported for a wide range of nonlinear dynamic economic models in discrete time (see, e.g., Agliari and Dieci, 2006; Agliari et al., 2007; and Dieci and Gallegati, 2011) and the mechanisms that lead to the appearance of new attractors or to the disappearance of existing ones are generally related to the occurrence of global bifurcations. What needs mentioning, especially for the economic interpretation of such phenomena, is the implication for the course of the underlying business dynamics. As is the case of the present model, the system will select among multiple long-run dynamic outcomes, which may include steady 

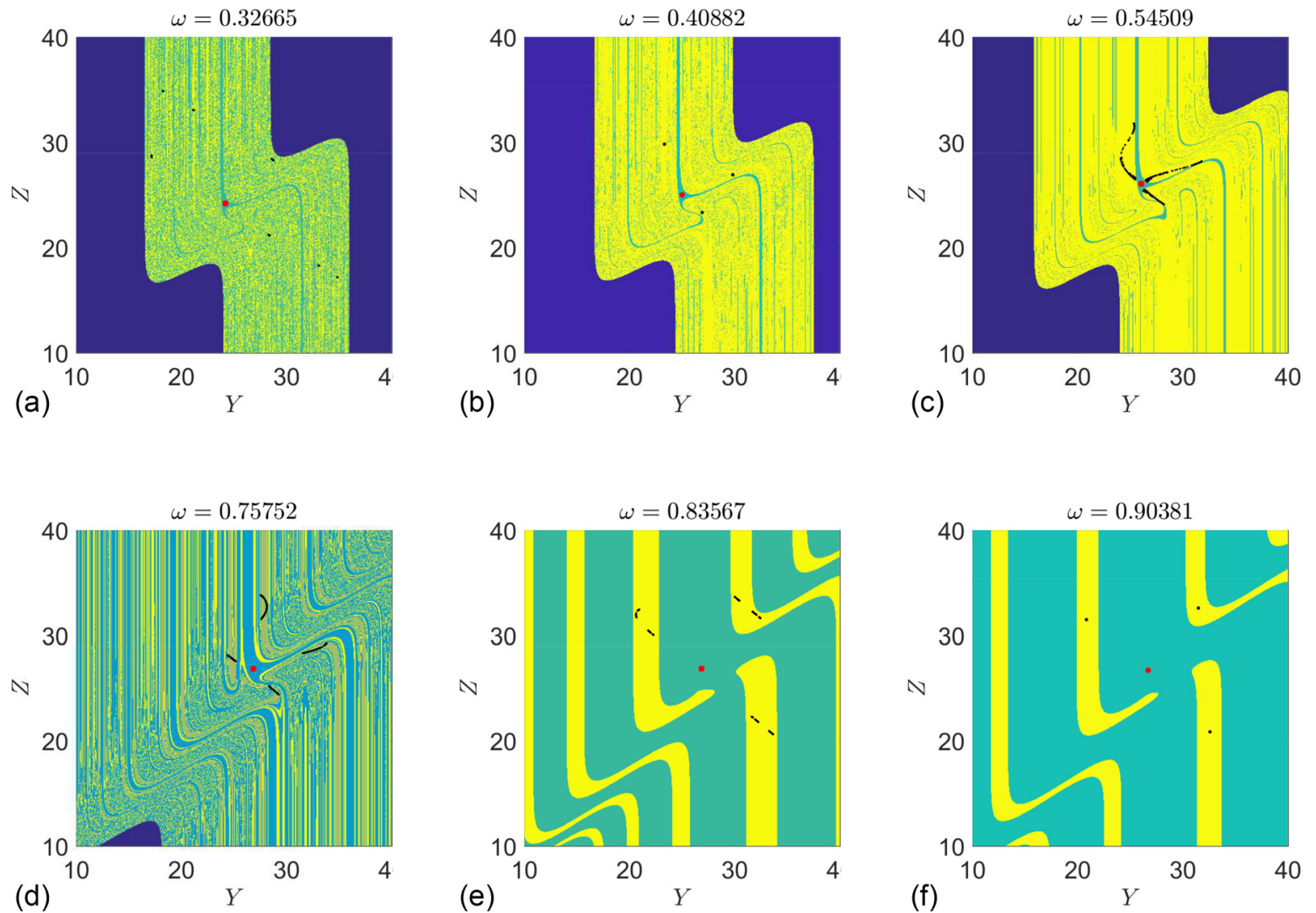

FIG. 3. Basins of attraction corresponding to some values of $\omega$ and for the parameter setting used for the simulation reported in Fig. 2(d). Color blue is used for unfeasible regions or divergence, color green for the basins of attraction of the steady state, and color yellow for the basins of coexisting attractors.

states and other types of attractors, e.g., periodic or chaotic attractors. In such cases, the representation of the basins of attraction, even in the presence of attractors with a relatively simple structure, helps in predicting which attractor will be selected by the economy. This is crucial for the long-run economic dynamics. Hence, the final outcome of the system may be totally uncertain when the initial condition is selected, in particular, the regions of the phase-plane and when the basins exhibit rather complicated structure: An uncertainty in the position of the phase space, or a shock, may generate the transition from convergence to one attractor to convergence to a different one. Moreover, thanks to such path dependence, the model is capable to encompass different stable scenarios for the same economic setting, accounting for the possibility that the same institutional and economic conditions can lead, depending on the past history, to different final outcomes (see Arthur, 1994).

To this end, in what follows we aim at understanding if the steady state, and/or an attractor coexisting with it, is sufficiently robust with respect to perturbations, namely, if, when trajectories are suitably close to an attractor, they can be driven toward another attractor by small nondeterministic fluctuations. In particular, we identify by $r_{\omega}$ the radius of the largest circular neighborhood of $\left(Y^{* *}, Y^{* *}\right)$ included in its basin of attraction, corresponding to the interaction degree $\omega$. Moreover, we identify by $B\left(Y^{* *}\right)$ the basin of attraction of $\left(Y^{* *}, Y^{* *}\right)$, while we use $\Gamma$ and $B(\Gamma)$ for any attractor coexisting with the steady state and for its basin, respectively.
In Fig. 3, we report $B\left(Y^{* *}\right)$ (in green color) and $B(\Gamma)$ (in yellow color) corresponding to $\omega \approx 0.32,0.545,0.76,0.84$. When $\omega \approx 0.32$, we have that $\Gamma$ is a seven pieces chaotic attractor whose basin $B(\Gamma)$ is very scrambled and mixed with $B\left(Y^{* *}\right)$ [see Fig. 3(a)]. We numerically checked that approximately the same number of initial data converges to each attractor. Moreover, $r_{0.32}$ is quite small, so even when trajectories are very close to the steady state, a small perturbation very likely can make them cross $B(\Gamma)$ and vice-versa.

In Figs. 3(b) and 3(c), we consider $\omega \approx 0.409$ and $\omega \approx 0.545$, respectively, both belonging to interval $(0.387$, 0.546), in which, looking at Fig. 2(d), a period-3 cycle [Fig. 3(b)] appears and evolves through a cascade of perioddoubling bifurcations toward a three pieces chaotic attractor [Fig. 3(c)]. We again have that the basins of attraction of the steady state and the coexisting three pieces attractor are scrambled, with $r_{0.545} \approx r_{0.32}$. Simulations suggest that only the ten percent of feasible initial data converge to the steady state. Finally, we notice that the three pieces chaotic attractor is close to become tangent to the boundary of $B\left(Y^{* *}\right)$ and to consequently disappear through a global bifurcation.

In Fig. 3(d), we report the complex structure of $B\left(Y^{* *}\right)$ and $B(\Gamma)$ when $\omega \approx 0.76 \in(0.715,0.758)$ and $\Gamma$ is a four pieces chaotic attractor coming from a cascade of perioddoublings of a period- 4 cycle. The main difference with the situation reported in Fig. 3(c) is that the probability to converge toward either the steady state or the chaotic attractor is reversed, as the number of initial data which converges toward the steady state results three times larger than that of 
those converging to the coexisting attractor. Moreover, $r_{0.75}>r_{0.545}$.

Finally, in Figs. 3(e) and 3(f) we consider $\omega \approx 0.84$ and $\omega \approx 0.90$, belonging to interval $(0.8,0.92)$, in which a chaotic attractor [Fig. 3(e)] is transformed into a period-3 cycle though a cascade of period-halvings [Fig. 3(f)]. Even if the structure of both $B\left(Y^{* *}\right)$ and $B(\Gamma)$ is more regular than in the previous cases, both basins are unconnected and a double number of initial data converge toward the steady state instead of $\Gamma$. In this last case, $r_{0.84}$ is larger than in the previous three examples, and the steady state is more robust with respect to perturbations.

The peculiar aspects common to the results described in Fig. 3 are that the basins of attraction can have very complex unconnected or scrambled structures, and even small perturbations can lead trajectories to unpredictably jump from $B\left(Y^{* *}\right)$ to $B(\Gamma)$ and vice-versa, even when we are very close to the steady state [for example, in Figs. 3(a)-3(d)]. Moreover, the ratio between the measures of $B\left(Y^{* *}\right)$ and $B(\Gamma)$ gives a piece of information about which basin is more frequently crossed by the trajectories, and, as a consequence, close to which attractor more likely they pass.

Keeping in mind the previous results and observations, in the last part of this section we investigate the qualitative properties of time series, in particular, depending on the degree of interaction between markets and on the accelerator. In this last set of simulations, we take into account a stochastic perturbation, to allow for exogenous shocks. To this end, we perturb both fundamentalists' and chartists' demands by a stochastic term. The demand placed by chartists is specified as

$$
D_{t}^{C}=e\left(P_{t}-F_{t}\right)+\varepsilon_{t}^{C},
$$

where $\varepsilon_{t}^{C}$ is introduced to capture part of the variety that can emerge in the technical analysis run by chartists. In fact, as argued by Murphy (1999), even if the spirit of technical analysts is to ride on a trend, there exist numberless different technical trading rules.

On the other hand, orders generated by fundamentalists read as

$$
D_{t}^{F}=f\left(F_{t}-P_{t}\right)+\varepsilon_{t}^{F},
$$

where $\varepsilon_{t}^{F}$ is introduced to capture the idea that it is quite difficult for investors to determine the fundamentals (in fact fundamental values may change over time due to real shocks), as already argued by Keynes (1936).

We assume that $\varepsilon_{t}^{C}$ and $\varepsilon_{t}^{F}$ are sequences of independent, identically distributed random normal variables, with zero mean and variance $\sigma_{F}^{2}$ and $\sigma_{C}^{2}$. Using the perturbed demands in (8) and acting as in Sec. II B we obtain

$$
P_{t}=(1-\omega) F+\omega d Y_{t}+\varepsilon_{t},
$$

where $\varepsilon_{t}$ is a sequence of independent, identically distributed random normal variables, with zero mean and variance $\sigma^{2}=\left(\sigma_{F}^{2}+\sigma_{C}^{2}\right) /(e-f)^{2}$.

Equation (27) is then used in (7) with policy (5) to obtain a stochastically perturbed version of model (14). The goal of this analysis is to demonstrate that a stochastic version of our model can generate realistic dynamics that are able to mimic several stylized facts of financial markets that, in turn, may affect the behavior of the real sector. Our analysis reveals that studying analytically the stability of the steady state become useful to understand its out-of-equilibrium behavior in the presence of noise. For related work on the intricate interplay between deterministic and random forces, see, for instance, Chiarella et al., 2011; He and Li, 2007; 2008; and Hommes 2013. In fact, it is worth mentioning that deterministic financial market models with interacting agents (see, e.g., Day and Huang, 1990; Lux, 1995; Brock and Hommes, 1997; and Chiarella et al., 2002) are able to produce intricate boom-bust cycles while stochastic model versions (see, e.g., Lux and Marchesi, 1999; Lux, 2009; Franke and Westerhoff, 2016; and Gaunersdorfer and Hommes, 2007) may match the statistical properties of financial markets in detail. Hence, thanks to their apparent explanatory power, models that have been being developed with interacting agents and sectors are increasingly used as tools for economic policy design (see Westerhoff and Franke (2013) for a survey).

In what follows, we study the times series of price returns, defined as

$$
R_{t}=100 \cdot\left(\log \left(P_{t}\right)-\log \left(P_{t-1}\right),\right.
$$

focusing, in particular, on the deviation from normality and volatility of their distribution. For each $\omega$ and $\gamma$, both $\operatorname{kurt}\left(R_{t}\right)$ and $\operatorname{vol}\left(R_{t}\right)$ are evaluated computing the average kurtosis and volatility obtained from 500 simulations with different sequences of $\varepsilon_{t}$.

In all the simulations reported in this section, we employ the same parameter setting as in the previous ones, and we set $\sigma=0.21$. In Fig. 4(a), we show the bifurcation diagram of $R_{t}$ under the effect of the stochastic shock, corresponding to the deterministic one of $Y_{t}$ reported in Fig. 2(d). A qualitative comparison of Figs. 4(a) and 2(d) highlights some differences between the deterministic and stochastically perturbed bifurcation diagrams. For $\omega<0.25$, from Fig. 4(a) we have no evidence of multistability, similar to the deterministic case, and the modest deviation from the steady state is just due to the small random fluctuations. When $\omega>0.25$, we can instead infer from Fig. 4(a) that we have at least an attractor coexisting with the steady state for any interaction degree. This is quite different from what is shown in Fig. 2(d), in which for some intervals of $\omega>0.25$ we again have that the steady state attracts all the initial data we considered. This different behavior can be understood by noticing that when introduce the stochastic component in the model, it is as if we are considering the first equation of (14) with a different constant $A$, depending on the actual value of $\varepsilon_{t}$. As a consequence, the resulting deterministic map can have different properties with respect to multistability than that corresponding to $\varepsilon_{t}=0$. Then, the behavior of the perturbed version of the model is even more complex than that reported in Figs. 2(c) and 2(d), and multistability can be introduced or destroyed depending on the perturbation, too. Both kurtosis [Fig. 4(b)] and volatility [Fig. 4(c)] behavior can be understood looking at the shape of the bifurcation diagram in Fig. 4(a). We recall that kurtosis measures how 


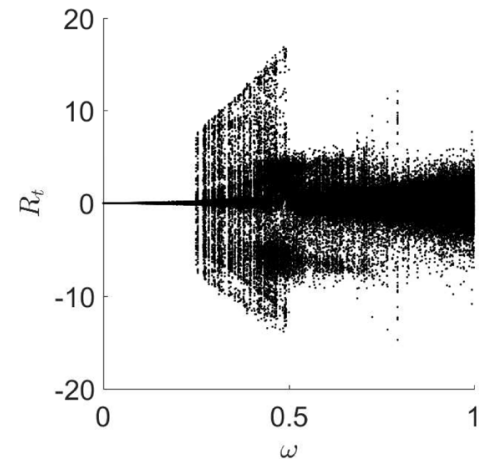

(a)

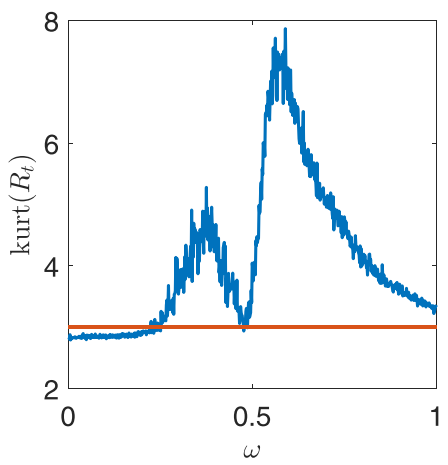

(b)

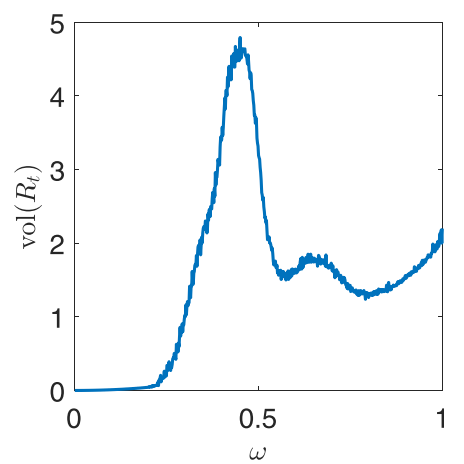

(c)

FIG. 4. (a) Bifurcation diagram of returns $R_{t}$ under the effect of stochastic shock $\varepsilon_{t}$. (b) Kurtosis, with orange line portraying the reference value 3 , representing normality. (c) Volatility. All the plots are obtained on varying $\omega$.

much fat are distribution's tails compared with those of a normal distribution. In this respect, to have a large kurtosis it is not sufficient to have price trajectories with large diameters but it is essential to have trajectories in which prices, which are far from their average, frequently occur.

When $\omega$ is suitably small, no coexistent attractors emerge, and the erratic trajectories are uniquely determined by the stochastic term, so the returns distribution reflects the normality of $\varepsilon_{t}$ and the volatility is very reduced. The large kurtosis for $\omega>0.25$ is essentially due to the presence of complex attractors. The non-monotonic behavior of $\operatorname{kurt}\left(R_{t}\right)$ is ascribed to the robustness of the emergence in the family of perturbed maps (14) of sequences of coexisting attractors. For example, for $\omega \in(0.25,0.5)$ we can infer from the bifurcation diagram in Fig. 4 that a family of coexisting attractors exists, and it is replaced by a different family for $\omega>0.5$, which illustrates the different kurtosis behavior for $\omega$ $\in(0.25,0.5)$ and for $\omega>0.5$. Moreover, for $\omega \approx 0.5$ the transition between the two different families of attractors makes multistability less robust, with a consequent reduced kurtosis. Moreover, the deviation from normality is more significant for $\omega \in(0.5,0.7)$ than for $\omega \in(0.25,0.5)$. This can be understood recalling the considerations we made about the basins of attraction reported in Figs. 3(a) and 3(b). Since for $\omega \in(0.5,0.7)$ the measure of the basin of $\Gamma, B(\Gamma)$, is larger than that of $B\left(Y^{* *}\right)$, trajectories are more likely to converge to the attractor $\Gamma$ with respect to the case when $\omega \in(0.25,0.5)$, in which the measure of the basins of the two attractors, $B(\Gamma)$ and $B\left(Y^{* *}\right)$ are similar. As a consequence, when $\omega \in(0.5,0.7)$, tails in returns' distributions are fatter. As $\omega$ further increases, recalling the comments concerning Figs. 3(c) and 3(d) about the effect of perturbations, the kurtosis decreases. The behavior of volatility is instead more closely connected to the diameter of the trajectories.

The key aspect is that, for most values of the interaction degree and of the accelerator, large kurtosis and volatility of price returns' distributions are the most common behavior, essentially fostered by the interactions between the two sides of the economy and the endogenous complexity of the model from the global stability point of view.

Another peculiar stylized fact of returns' times series is the volatility clustering, namely, the occurrence of several consecutive returns characterized by large volatility alternated with several consecutive returns characterized by small volatility. This is graphically evident from Fig. 5(a), in which a typical example of time series of returns, obtained for the parameter setting and the stochastic perturbations used for Fig. 4, is reported. If, at each time period $t$, we compute the average volatility considering time periods $t-30, t-29, \ldots, t$ (i.e., the average moving volatility over a window of 30 time periods), we can see that intervals characterized by large and small volatility alternates [Fig. 5(b)]. Finally, volatility clustering is highlighted by the typical strongly positive, slowly decreasing autocorrelation coefficients of absolute returns, reported in Fig. 5(c).

We remark that if we slightly change the standard deviation of $\varepsilon_{t}$, all the previous results remain qualitatively the

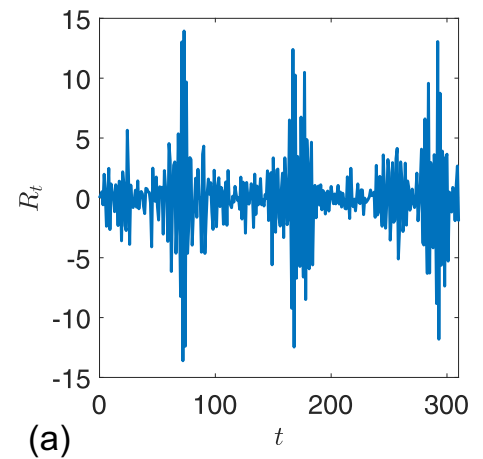

(a)

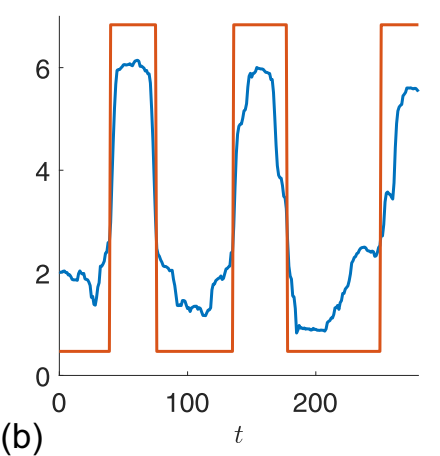

(b)

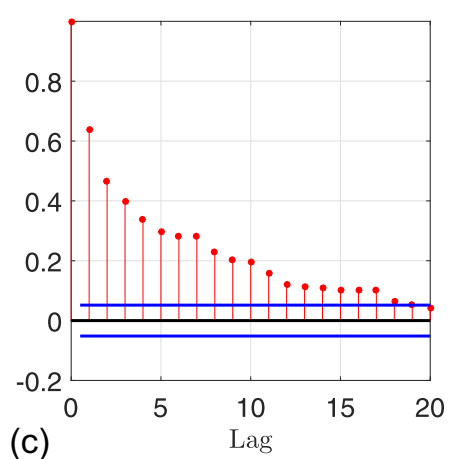

(c)

FIG. 5. (a) Time series of returns. (b) Moving volatility on a window of 30 time periods of the returns. The orange line shows intervals in which volatility is larger or smaller than the volatility computed considering the whole time series. (c) Autocorrelation of absolute returns $\left|R_{t}\right|$.. 
same. Indeed, as $\sigma \approx 0$, the deterministic component is the dominant one and the results are no more significant, as well as if we keep increasing $\sigma$. In this case, the shock becomes dominant and the returns distribution become increasingly close to a normal one, even if volatility increases. Finally, even if we just reported results concerning a family of simulations related to a unique parameter setting, we numerically checked that the emergence of coexistence phenomena and of the consequent stylized facts in the time series is robust. Indeed, they can arise for different (either smaller or larger) values of $\omega$ with respect to those reported in the previous simulations, but results and considerations remains qualitatively the same.

Overall, we can state that by adding a stochastic shock to the speculators' demand, which reflects certain withingroup heterogeneity, we have been able to replicate several statistical properties of real financial markets. The resulting outcomes can be seen as complementary to the deterministic analysis, providing a broader macroscopic picture of the emergent properties that may arise when connecting the two (real and financial) subsystems.

\section{CONCLUDING REMARKS}

In this paper, we proposed a model belonging to the strand of literature on the interactions between real and financial markets (see, e.g., Westerhoff, 2012 and Naimzada and Pireddu, 2015). The real side of the economy, which is described within a nonlinear multiplier-accelerator framework, where the nonlinearity is ascribed to the investment function, interacts with a financial market with heterogeneous speculators. This allowed us to focus on the role of the degree of interaction between the two markets, considering two different well-known fiscal policy rules and studying their capability to stabilize the course of the economy, leading it toward the desired output steady state and how the accelerator influence such dynamics. By means of both analytical and numerical tools, we carried on the study at three levels: a first static investigation of the possible steady states and how they vary, the analysis of their local stability, and the possible emergence of multistability phenomena. The static analysis showed that a suitable degree of interaction may generate a higher income level with respect to when they are independent, both for the level-adjusting and the trend-offsetting policy rules. The beneficial effect of the degree of interaction seems to emerge also from the local stability analysis, with a possible stabilizing effect for suitable accelerator values. However, deepening the analysis to a global level reveals that multistability phenomena may occur even when the national income steady state is locally stable, independently of the interaction degree. As the interaction degree increases, the stable steady state can coexist with complex attractors, giving rise to endogenous erratic fluctuations. This level of investigation is essential to reproduce the recurrent boom-bust sequences observed in the real financial markets together with several stylized facts regarding stock prices and their return (and output as well), such as positive autocorrelation, volatility clustering, and nonnormal distribution characterized by a high kurtosis and fat tails. We plan to extend the research about real and financial interacting markets in several ways. A first way is to consider the introduction of an adaptive learning mechanism through which agents can switch between the demand they place in the market according to a performance measure, to see how this increase or reduce the overall dynamical complexity and improve the qualitative description of the real situation. Another possibility is to take into account money or other financial asset as well as to investigate the interaction occurring by the introduction of another speculative market, as the housing market. Finally, more sophisticated approaches in modelling the time frameworks of either or both the real and the financial markets can be considered. The modelling of the present economic setting would benefit from a more refined and sophisticated time description, in particular, that take into account the different speed of the adjustment mechanisms of prices and agents of real and financial sectors (the latter is usually faster). In fact, the dynamics of financial markets are likely to be very different from those of the real markets: Although this argument seems nowadays plain, it is also backed empirically in the work by Aoki and Yoshikawa (2011), who find that time series of real economic data do not share the power law distribution of financial markets, implying that the latter are characterized by higher economic activity. Additionally, Lengnick and Wohltmann (2013) combine a simple agentbased model of financial markets with a standard New Keynesian macroeconomic model considering two different time scales for the two sectors and bringing them together.

From the mathematical viewpoint, this can be realized in several ways. Besides the classical approach based on differential equations, we can mention the choice of delay differential equations (as pursued in Matsumoto and Szidarovszky, 2015 and Gori et al., 2016), in which introducing distinct delays for the real and the financial markets would allow studying the effect of asynchronous decision timing. Finally, as in Cavalli and Naimzada (2016) and Lamantia and Radi (2015), another approach relies on hybrid dynamical systems, in which discrete difference and continuous differential equations are, respectively, used to model the real and the financial market.

\section{ACKNOWLEDGMENTS}

The authors wish to thank the Editor and two anonymous referees for useful comments and suggestions. The usual caveats apply.

Agliari, A. and Dieci, R., Coexistence of Attractors and Homoclinic Loops in a Kaldor-like Business Cycle Model, Business Cycle Dynamics: Models and Tools (Springer-Verlag, 2006).

Agliari, A., Dieci, R., and Gardini, L., "Homoclinic tangles in a kaldor-like business cycle model," J. Econ. Behav. Organ. 62, 324-347 (2007).

Aoki, M. and Yoshikawa, H., Reconstructing Macroeconomics: A Perspective from Statistical Physics and Combinatorial Stochastic Processes (Cambridge University Press, 2011).

Arthur, W. B., Increasing Returns and Path Dependence in the Economy (University of Michigan Press, 1994).

Bask, M., "Asset price misalignments and monetary policy," Int. J. Finance Econ. 17, 221-241 (2012). 
Bischi, G. I., Dieci, R., Rodano, G., and Saltari, E., "Multiple attractors and global bifurcations in a Kaldor-type business cycle model," J. Evol. Econ. 11, 527-554 (2001).

Brock, W. A. and Hommes, C. H., "A rational route to randomness," Econometrica 65, 1059-1095 (1997)

Cavalli, F. and Naimzada, A., "A multiscale time model with piecewise constant argument for a boundedly rational monopolist," J. Differ. Eq. Appl. 22, 1480-1489 (2016).

Charpe, M., Flaschel, P., Hartmann, F., and Proaño, C., "Stabilizing an unstable economy: Fiscal and monetary policy, stocks, and the term structure of interest rates," Econ. Model. 28, 2129-2136 (2011).

Chen, X., Kontonikas, A., and Montagnoli, A., "Asset prices, credit and the business cycle," Econ. Lett. 117, 857-861 (2012).

Chiarella, C., Dieci, R., and Gardini, L., "Speculative behavior and complex asset price dynamics: A global analysis," J. Econ. Behav. Organ. 49, 173-197 (2002).

Chiarella, C., He, X. Z., and Zheng, M., "An analysis of the effect of noise in a heterogeneous agent financial market model," J. Econ. Dyn. Control 35, 148-162 (2011).

Day, R. H. and Chen, P., Nonlinear Dynamics and Evolutionary Economics (Oxford University Press, 1993).

Day, R. H. and Huang, W., "Bulls, bears and market sheep," J. Econ. Behav. Organ. 14, 299-329 (1990).

Dieci, R. and Gallegati, M., "Multiple attractors and business fluctuations in a nonlinear macro-model with equity rationing," Math. Comp. Model. 53, 1298-1309 (2011).

Franke, R. and Westerhoff, F., "Why a simple herding model may generate the stylized facts of daily returns: Explanation and estimation," J. Econ. Interact. Coord. 11, 1-34 (2016).

Gaunersdorfer, A. and Hommes, C., "A nonlinear structural model for volatility clustering," in Long Memory in Economics (Springer Berlin Heidelberg, 2007), pp. 265-288.

Goodwin, R. M., "The nonlinear accelerator and the persistence of business cycles," Econometrica 19, 1-17 (1951).

Gori, L., Guerrini, L., and Sodini, M., "Disequilibrium dynamics in a keynesian model with time delays," Commun. Nonlinear Sci. Numer. Simul. (in press).

He, X. Z. and Li, Y., "Power-law behavior, heterogeneity, and trend chasing," J. Econ. Dyn. Control 31, 3396-3426 (2007).

$\mathrm{He}, \mathrm{X}$. Z. and Li, Y., "Heterogeneity, convergence, and autocorrelations," Quant. Finance 8, 59-79 (2008).

Hicks, J. R., A Contribution to the Theory of the Trade Cycle (Clarendon Press, Oxford, 1950).

Hommes, C., Behavioral Rationality and Heterogeneous Expectations in Complex Economic Systems (Cambridge University Press, 2013).

Hommes, C. H., "A reconsideration of Hicks' non-linear trade cycle model," Struct. Change Econ. D 6, 435-459 (1995).

Kaldor, N., "A model of the trade cycle," Econ. J. 50, 78-92 (1940).
Kalecki, M., "A macrodynamic theory of business cycles," Econometrica 3, 327-344 (1935).

Keynes, J., The General Theory of Employment, Interest, and Money (The Easton Press, 1936).

Kontonikas, A. and Montagnoli, A., "Optimal monetary policy and asset price misalignments," Scott. J. Politi. Econ. 53, 636-654 (2006).

Lamantia, F. and Radi, D., "Exploitation of renewable resources with differentiated technologies: An evolutionary analysis," Math. Comp. Simul. 108, 155-174 (2015).

Lengnick, M. and Wohltmann, H. W., "Agent-based financial markets and New Keynesian macroeconomics: A synthesis,” J. Econ. Interact. Coord. 8, 1-32 (2013).

Lux, T., "Herd behavior, bubbles and crashes," Econ. J. 105, 881-896 (1995).

Lux, T., "Stochastic behavioral asset-pricing models and the stylized facts," in Handbook of Financial Markets: Dynamics and Evolution (NorthHolland, Amsterdam, 2009), pp. 161-216.

Lux, T. and Marchesi, M., "Scaling and criticality in a stochastic multi-agent model of a financial market," Nature 397, 498-500 (1999).

Matsumoto, A., "Note on Goodwin's 1951 nonlinear accelerator model with an investment delay," J. Econ. Dyn. Control 33, 832-842 (2009).

Matsumoto, A. and Szidarovszky, F., "Nonlinear multiplier-accelerator model with investment and consumption delays," Struct. Change Econ. D. 33, 1-9 (2015).

Medio, A. and Lines, M., Nonlinear Dynamical Systems in Economics (Springer, 2005).

Murphy, J., Technical Analysis of Financial Markets (New York Institute of Finance, 1999).

Naimzada, A. and Pireddu, M., "Dynamic behavior of product and stock markets with a varying degree of interaction," Econ. Model. 41, 191-197 (2014).

Naimzada, A. and Pireddu, M., "Real and financial interacting markets: A behavioral macro-model," Chaos Soliton Fract. 77, 111-131 (2015).

Naimzada, A. and Pecora, N., "Dynamics of a multiplier-accelerator model with nonlinear investment function," Nonlinear Dyn. 88, 1147-1161 (2017).

Puu, T., Gardini, L., and Sushko, I., "A Hicksian multiplier-accelerator model with floor determined by capital stock," J. Econ. Behav. Organ. 56, 331-348 (2005).

Samuelson, P. A., "Interactions between the multiplier analysis and the principle of acceleration," Rev. Econ. Stat. 21, 75-78 (1939).

Sushko, I., Gardini, L., and Puu, T., "Regular and chaotic growth in a Hicksian floor/ceiling model,” J. Econ. Behav. Organ. 75, 77-94 (2010).

Westerhoff, F., "Interactions between the real economy and the stock market: A simple agent-based approach," Discrete Dyn. Nat. Soc. 2012, 504840 .

Westerhoff, F. and Franke, R., "Agent-based models for economic policy design," in Handbook on Computational Economics and Finance (Oxford University Press, 2013). 